\title{
Philosophiques
}

\section{Conscience de soi et langage intérieur : quelques spéculations}

\section{Alain Morin et James Everett}

Volume 17, numéro 2, automne 1990

URI : https://id.erudit.org/iderudit/027124ar

DOI : https://doi.org/10.7202/027124ar

Aller au sommaire du numéro

\section{Éditeur(s)}

Société de philosophie du Québec

ISSN

0316-2923 (imprimé)

1492-1391 (numérique)

Découvrir la revue

Citer cette note

Morin, A. \& Everett, J. (1990). Conscience de soi et langage intérieur : quelques spéculations. Philosophiques, 17(2), 169-188. https://doi.org/10.7202/027124ar

\section{Résumé de l'article}

Ce texte propose une définition de la conscience de soi et explique en quoi cette capacité naît du monde social. Il est postulé que ce dernier permet un mouvement de recul - une "distanciation » - par rapport à soi, et que le cerveau reproduit ce mouvement grâce à certains processus cognitifs qui en ont été imprimés. Parmi ceux-ci, on retrouve le langage intérieur, qui, par analogie, agirait comme un miroir interne capable de confronter l'expérience subjective à elle-même; de cette confrontation naîtrait le soi. Un argument est présenté en faveur de la supériorité du langage intérieur sur d'autres processus cognitifs impliqués dans la conscience de soi. Le problème de la conscience de soi chez les primates est abordé, et l'article conclut sur diverses réflexions ayant trait à la schizophrénie, la prière, la méditation, et les drogues psychodysleptiques. 


\title{
NOTE DE RECHERCHE
}

\section{CONSCIENCE DE SOI ET LANGAGE INTÉRIEUR : QUELQUES SPÉCULATIONS}

\author{
par Alain Morin et James Everett
}

\begin{abstract}
RÉsumÉ. Ce texte propose une définition de la conscience de soi et explique en quoi cette capacité naît du monde social. Il est postulé que ce dernier permet un mouvement de recul - une *distanciation * - par rapport à soi, et que le cerveau reproduit ce mouvement grâce à certains processus cognitifs qui en ont été imprimés. Parmi ceux-ci, on retrouve le langage intérieur, qui, par analogie, agirait comme un miroir interne capable de confronter l'expérience subjective à elle-même; de cette confrontation naîtrait le soi. Un argument est présenté en faveur de la supériorité du langage intérieur sur d'autres processus cognitifs impliqués dans la conscience de soi. Le problème de la conscience de soi chez les primates est abordé, et l'article conclut sur diverses réflexions ayant trait à la schizophrénie, la prière, la méditation, et les drogues psychodysleptiques.
\end{abstract}

ABSTRACT. This text proposes a definition of self-awareness and explains its social origin. It is postulated that the social milieu permits a movement to a more objective perspective for selfperception, and that this movement is then reproduced in the brain by specific cognitive processes. It is suggested that inner specch represents one such cognitive processes, which acts like a mirror to reflect subjective experience back upon itself; the self would be generated by this reflective activity. It is argued that inner specch has a pre-eminent position among the cognitive processes implicated in self-awareness. The problem of self-awareness in primates is discussed, and the article concludes with ideas concerning schizophrenia, prayer, meditation, and psychodysleptic drugs. 
Qu'est-ce que la conscience de soi, et quel rapport entretient-elle avec l'expérience subjective? Qu'est-ce qui rend possible l'apparition de cette capacité, et avec elle, vraisemblablement, du soi? En quoi le langage intérieur est-il impliqué, plus étroitement que tout autre processus cognitif, dans l'auto-observation? Les animaux sont-ils conscients d'eux-mêmes malgré l'absence apparente chez eux d'une forme de communication intra-personnelle?

Telles sont les principales questions que nous allons explorer de façon spéculative dans le présent article, sans soucis à ce point-ci d'amener sur un terrain empirique ces considérations. ${ }^{1}$ De même, le lecteur ne doit pas espérer retrouver un relevé de littérature exhaustif sur le sujet, et encore moins des conclusions fermes. Nous avons plutôt saisi l'opportunité ici d'articuler en un tout cohérent des idées personnelles auparavant disparates.

La littérature scientifique et philosophique regorge de publications dont les titres contiennent le mot consciousness. Trop souvent, ce terme n'y est tout simplement pas défini ${ }^{2}$; certains auteurs prêtent à ce mot une signification très large ${ }^{3}$, et d'autres, une signification très précise. ${ }^{4}$ Une confusion courante règne lorsqu'il s'agit de distinguer entre elles les notions de consciousness et de self-consciousness, où le premier terme est souvent pris comme signifiant le second. ${ }^{5}$ De plus - ce qui ne fait qu'aggraver une situation déjà gênante - le terme français conscience réfère généralement au terme anglais self-consciousness, et le terme anglais consciousness, au terme français conscience de soi. Et tout ceci sans parler des termes mind, self, awareness, self-awareness, introspection, self-observation, etc., équiva-

1. Pour un traitement expérimental de la question du rapport entre la conscience de soi et le langage intérieur, se référer à A. Morin et J. Everett (1990). * Inner speech as a mediator of self-awareness, self-consciousness and self-knowledge : an hypothesis *. New Ideas in Psycbology, sous presse.

2. Un exemple typique est l'article de R. Puccetti (1977). « Bilateral organization of consciousness in man *. Annals of the New York Academy of Sciences, 299, 448-458.

3. Notamment S.J. Dimond (1976). «Brain circuits for consciousness *. Brain and Bebavior Evolution, 13, 376-395.

4. Notamment G.G. Gallup, Jr. (1985). «Do minds exist in species other than our own? * Neuroscience and Behavioral Reviews, 9, 631-641

5. L.S. Vygotsky a tendance à commettre cette erreur dans Thought and Language (1962; New York: John Wiley). 
lents nébuleux ou hyper-sophistiqués de consciousness et de self-consciousness.

Face à un tel capharnaüm, une définition claire de ce qu'est la conscience de soi, même si celle-ci risque d'être arbitraire, s'impose. ${ }^{6}$ Tout d'abord, il s'avère impératif de ne pas confondre conscience avec conscience de soi. La conscience représente un état d'éveil et d'activation physiologique cérébrale opposé à l'inconscience, état caractéristique du sommeil ou du coma. ${ }^{7}$ Généralement, la conscience précède - est un prérequis à - l'expérience subjective, c'est-à-dire nos perceptions, émotions, sensations, pensées, souvenirs, images mentales, etc.; mais tout organisme conscient (éveillé) n'est pas nécessairement connaissant de l'expérience subjective dont il est le sujet (conscient de soi). Plus précisément, être * capable de conscience * signifie être capable de traiter l'information en provenance de l'environnement - être en état d'éveil. Être «capable de conscience de soi * (ou : être capable de prendre connaissance du contenu de son expérience subjective) signifie pouvoir traiter l'information en provenance de soi.

En fait, une portion importante de notre activité mentale se déroule à notre insu. ${ }^{8}$ C'est ce qui se produit lorsqu'une sensation par exemple n'est pas « ressentie * mais est bien réelle puisque génératrice d'un comportement. Ainsi, une sensation d'irritation peut être provoquée par le port de nos vêtements; elle produira un comportement inconscient et automatique consistant à se gratter. De la même façon, on peut dire qu'il nous arrive fréquemment de percevoir quelque chose sans le "voir" : notre cerveau enregistre une image précise, mais notre attention étant dirigée sur autre chose, il n'y a pas perception consciente de l'objet nous "fixons dans le vide*. Le même phénomène se répète lorsque nous sommes sujets d'émotions particulièrement intenses, comme la colère: pendant une brève période de temps, une expérience subjective à laquelle nous n'avons pas accès a lieu, et ce n'est qu'après coup qu'il devient possible pour nous d'observer les comportements qu'elle a

6. T. Natsoulas (1983) serait définitivement d'accord avec nous sur ce point, ainsi que sur notre constatation de l'existence d'une confusion dans la littérature. Cf. *Concepts of consciousness*, The Journal of Mind and Bebavior, 4(1), $13-59$

7. Cf. T. Natsoulas (1978), "Consciousness" American Psychologist, 34(2), 906. 914 ; J. DeWitt (1975), *Consciousness, mind and self: the implications of split-brain studies *, British Journal of the Pbilosophy of Sciences, 26, 11-47.

8. R.J. Davidson (1980), "Consciousness and information processing : a biocognitive perspective *. Dans J.M. Davidson et R.J. Davidson (Eds.), The Psychobiology of Consciousness. New York: Plenum Press. 
générés (d'où le rapport verbal typique «je ne savais pas ce que je faisais !...* lorsqu'on s'est brutalement fâché).

On peut donc dire, compte tenu de ce qui précède, que tout organisme sujet à une expérience subjective est conscient; toutefois, seul le cerveau humain possède avec certitude la capacité de prendre connaissance du contenu de son expérience subjective ainsi que de cet état de fait c'est la conscience de soi. ${ }^{9}$

Nous serions portés à croire avec $\operatorname{Prado}^{10}$ que la conscience de soi comporte au moins deux dimensions ou niveaux :

1 un accès à, une connaissance de, nos états mentaux ("awareness of one's mental states");

2 une "vision" de soi comme étant connaissant de quelque chose - une prise de conscience de soi en train de prendre connaissance du contenu de son expérience subjective ("awareness of ourselves as conscious of something"). ${ }^{11}$

Notons qu'il est très important de ne pas concevoir ces deux dimensions comme étant deux états indépendants et qualitativement différents : il s'agit vraisemblablement de deux manifestations - la seconde étant plus accentuée que la première - d'un seul et même processus, où «mécanisme». Cet aspect du problème sera approfondi plus loin.

9. En psychologie sociale, la conscience de soi est généralement définie comme la capacité chez un organisme de devenir l'objet de sa propre attention (Duval et Wicklund, 1972). Buss (1980), notamment, a reconnu et démontré empiriquement que notre attention peut être dirigée plus ou moins fréquemment sur des aspects privés ou publics de soi, d'où une conscience de soi privée qui réfere à l'attention portée aux sentiments et pensées personnelles, et une conscience de so i publique, c'est-à-dire, une conscience de soi en tant qu'objet social. Notre définition recouvre la notion de conscience de soi privée; celle de conscience de soi publique sera volontairement négligee dans cet article. S. Duval et R.A Wicklund (1972), A Theory of Objective Self Awareness, New York : Academic Press. A.H. Buss (1980), Self-Consciousness and Social Anxiety. San Francisco Freeman.

10. G.G. Prado (1977). « Reference and consciousness *, Australasian Journal of Pbilosopby, 55(1), 22-26.

11. Une autre façon de concevoir ces deux dimensions s'inspire de Van Gulick : *A conscious mental state may be either a mental state of wbicb one is conscious (i.e., a mental state that is the object of self-awareness) or a state of being conscious of some mental state (i.e., a state of self-awareness which has a mental state as its object)*. R. Van Gulick (1985). "Conscious wants and self-awareness *. The Bebavioral and Brain Sciences, 8, 555-556. 


\section{II}

Il convient pour l'instant de poser la question fondamentale qui guide la présente réflexion : comment naît la conscience de soi? Qu'est-ce qui rend possible l'apparition de cette capacité?

C'est d'abord et avant tout le monde social qu'il faut accréditer ici. Mead ${ }^{12}$ par exemple, a suggéré que le milieu social permettait la formation graduelle d'une idée de ce que l'on est en confrontant l'individu à des façons différentes de penser, de sentir et d'agir - en le forçant à emprunter le point de vue d'autrui, ce qui met en relief ses propres caractéristiques. Cooley ${ }^{13}$ a comparé autrui à des miroirs, où l'individu, en considérant les commentaires et les réactions des autres face à lui, développerait une image de soi. Piaget ${ }^{14}$ recoupe cette analyse en proposant que la pensée humaine, égocentrique au départ, serait inapte à percevoir ses propres contradictions, à vérifier les affirmations qu'elle porte, ou à s'assurer qu'elle se fait bien comprendre par autrui. C'est la confrontation répétée à autrui qui amènerait peu à peu l'enfant à l'au to-critique et à la conscience de soi.

Tout environnement social suffisamment complexe semble donc être la racine d'un phénomène majeur où chaque membre de cet environnement peut représenter un miroir pour les autres, et vice-versa. Approfondissons cette idée.

Il faut d'abord comprendre que l'individu reçoit de sa confrontation avec autrui au moins trois types d'auto-information. Il y a ces aspects de son comportement et de son activité mentale qui, étant différents de ceux des autres, lui «sautent aux yeux»- d'où une prise de connaissance de quelque chose qui était implicite auparavant; il y a ces aspects qu'autrui se contente d'observer et de souligner, permettant ainsi à l'individu de se percevoir sous un angle lui étant autrement inaccessible ; et il y a la simple attention que porte sur lui autrui, l'incitant à s'auto-observer. Ensuite, une multitude de situations non sociales renseignent aussi - et de la même façon - l'individu à son propos. Par exemple, le cinéma et la télévision nous montrent des

12. G.H. Mead (1934), Mind, Self, and Society. Chicago: University of Chicago Press

13. C.H. Cooley (1902), Human Nature and The Social Order. New York: Scribners

14. J. Piaget (1924), Le jugement et le raisonnement chezl'enfant. Paris : Delachaux et Niestlé 
comportements différents des nôtres ; le vidéo portatif nous permet - bien plus que le simple miroir - d'observer ses propres comportements; la lecture nous confronte à des façons de penser différentes de la nôtre; et même la relecture de nos propres écrits (ou de toute autre forme de création et d'expression) avec un recul temporel met en relief notre "style*.

Dans tous ces cas, il y a "redondance * d'une information particulière ${ }^{15}$ : qu'il s'agisse d'un comportement ou d'un élément donné de l'expérience subjective, l'individu, en plus de l'émettre ou de le vivre intérieurement, est en position de le voir. D'où un recul, une distanciation ${ }^{16}$ face à soi-même.

Si le monde social doit être crédité dans l'avènement et le maintien de la conscience de soi, nous proposons aussi qu'il en va de même pour certain processus cognitifs (des "outils" mentaux faisant partie de l'expérience subjective mais étant spécifiquement impliqués dans l'acquisition et le traitement de l'information) dont la fonction est de reproduire sur un plan intra-psycbique ce qui se passe tout d'abord sur un plan social. Luria ${ }^{17}$ a déjà proposé que l'organisation de nos fonctions psychologiques supérieures a été littéralement façonnée, modulée, par l'environnement social dans lequel le cerveau a évolué. D'où la proposition suivante, relative à la conscience de soi : le cerveau a été graduellement «imprimé " par le mouvement de recul par rapport à soi qui origine du monde social, et est ainsi devenu le médiateur de ce mouvement par le biais de processus purement cognitifs.

Dans cette perspective, l'environnement social serait nécessaire à l'ébauche de la conscience de soi, mais insuffisant pour qu'un degré réellement humain en soit atteint. Tout comme un désir de communication prenant racine dans le monde social doit, pour se concrétiser, être médiatisé par des processus linguistiques de nature cognitive, de la même façon, la conscience de soi devrait, une fois initiée par l'environnement social, être "reproduite " par des processus cognitifs précis.

15. Cf. Routtenberg à ce propos. A. Routtenberg (1980), « Redundancy in the central nervous system*. Dans J.M. Davidson et R.J. Davidson (Eds.), The Psycbobiology of Consciousness, New York: Plenum Press.

16. Bien que ce mot ne soit pas français, nous l'utiliserons ici, faute de mieux.

17. A.R. Luria (1978), Les fonctions corticales supérieures de l'bomme, Paris, Presses Universitaires de France 


\section{III}

Comment le cerveau s'y prend-il pour opérer une "distanciation", un recul par rapport à lui-même? Il s'agit là d'une question fort embarrassante qu'il convient peut-être mieux ici de traiter avec une analogie, faute d'explication solide. Nous proposons l'analogie suivante : le cerveau dispose de miroirs internes permettant à l'expérience subjective qu'il génère de se détacher d'elle-même. Ces miroirs seraient des processus cognitifs capables de "dédoubler", en tout ou en partie et à un moment donné, l'expérience subjective, créant ainsi une redondance au sein du système. Or, parmi ces processus, le plus apte, le plus précis à expliciter le contenu de l'expérience subjective à l'organisme est, selon nous, le langage intérieur - ou, en termes opérationnels, l'activité de se parler à soi-même à propos de soi. ${ }^{18}$ Nous y reviendrons sous peu.

Pour l'instant, rappelons que la conscience de soi comporte au moins deux dimensions : une prise de connaissance du contenu de son expérience subjective, et une prise de conscience de cet état de fait où le sujet de l'expérience subjective se voit s'auto-observer.

Mais qui est le sujet de l'expérience subjective? Qui devient en mesure de s'auto-observer? C'est ici que le délicat problème du «self» (le soi) doit être abordé.

Notre position veut que le soi (la personne humaine en tant qu'elle a conscience d'elle-même, et qu'elle est à la fois sujet et objet de la pensée) naisse de - ou tout au moins, se manifeste suite ̀̀ - la «distanciation» de l'expérience subjective avec elle-même. Il est difficile d'imaginer qu'un organisme littéralement "collé" à l'expérience subjective qu'il vit puisse réaliser qu'il en est le sujet. ${ }^{19}$ L'expérience subjective représentant certainement une des plus impor-

18. On se parle à soi-même à propos d'une foule de choses. Le langage intérieur sert donc plusieurs fonctions cognitives, dont une des plus importantes est certainement l'auto-régulation (cf. Zivin pour un relevé de littérature). Dans cet article, nous ne discuterons que du role du langage intérieur dans l'acquisition d'auto-informations - nous ferons toujours référence à l'activité de se parler à soi-même a propos de soi. G. Zivin [Ed.] (1979). The Development of Self-Regulation Through Private Speecb. New York: Wiley

19. Johnstone note «(...) qu'une personne peur être consciente de quelque chose seulement si une distance est créée entre elle et cette chose. (...) Dans une immersion complète dans l'experience subjective, il ne peut y avoir un sens de possession de cette expérience. H.W. Johnstone (1970). The Problem of The Self. Pennsylvania State University Press. [Citation : p. 106 ; cette citation, ainsi que toutes les autres dans cet article, sont des traductions libres] 
tantes parties de ce qu'est l'organisme (au même titre que son corps et que ses comportements), cela signifie qu'avant la "distanciation ", l'organisme n'est pas en mesure d'observer une part substantielle de lui-même. Et si la capacité d'auto-observation (dimension 1 de la conscience de soi) constitue un prérequis à la réalisation de son existence (dimension 2 de la conscience de soi), alors cet organisme n'est pas connaissant de son existence avant la "distanciation" d'une partie fondamentale de lui-même avec celle-ci.

Donc, le phénomène de «distanciation» postulé ici confronterait par redondance l'expérience subjective avec elle-même et le reste de l'organisme, et de cette confrontation apparaîtrait la possibilité pour un soi non existant avant cette "distanciation» de s'auto-observer. Empruntons une analogie pour clarifier ce qui précède.

Imaginons un individu qui $n$ 'a jamais été confronté à une surface réfléchissante, où, pour les besoins de la cause, les caractéristiques morphologiques du corps de cette personne représentent des éléments précis de l'expérience subjective. Dans un premier temps (temps A), cet individu est placé en face et très près d'un miroir, de sorte qu'il ne puisse observer que des éléments épars de sa physionomie (son nez, son œil, son épaule droite, etc.). Petit à petit, on écarte l'observateur du miroir afin qu'encore plus d'éléments puissent être observés simultanément, jusqu'au moment (temps B) où cette distance séparant l'observateur de la chose observée soit suffisamment grande pour qu'il saisisse que ce qu'il observe est lui-même - prenant ainsi conscience de son existence. À ce point, il s'observera d'une façon très différente : lorsqu'il examinera son nez par exemple, il saura qu'il lui appartient; et lorsqu'il percevra sa réflexion dans son ensemble, il se verra s'observer.

Décomposons en nos termes les dimensions 1 et 2 de la conscience de soi et voyons en quoi cette analogie peut nous aider à comprendre les rapports qui les unissent.

Dimension 1 - identification d'éléments constituant

l'expérience subjective (l'organisme,

temps A

en plus de vivre une expérience subjective,

est capable de l'identifier);

Dimension 2 - (a) identification d'éléments constituant son expérience subjective (l'organisme, en plus de vivre et d'identifier une expérience subjective, sait qu'elle est sienne);

temps $\mathrm{B}$

(b) observation de soi en train

d'identifier des éléments de son

expérience subjective. 
La «distanciation" (d'abord sociale, et ensuite, parallèlement, cognitive) de l'expérience subjective avec ellemême s'effectue vraisemblablement en deux temps, où l'organisme commence par identifier ce qui se passe en lui, pour pouvoir ensuite percevoir simultanément suffisamment d'éléments de lui-même pour se voir et comprendre que ce qu'il observe est lui-même. À ce moment seulement, il comprendra aussi que ce qu'il vit intérieurement lui appartient.

N'oublions pas que l'observateur (le soi, une fois le point $B$ atteint) et la chose observée (l'expérience subjective) sont nécessairement identiques. Par conséquent, il est fort tentant d'en conclure que le soi est, en grande partie tout au moins, l'expérience subjective. Cette idée est très attirante puisqu'elle encourage une vision moins dualiste du problème: nous n'avons pas besoin d'identifier le soi à une entité abstraite capable de "voir» ce qui se passe en nous, ou pis encore, à une entité de nature immatérielle. Le soi serait, dans notre optique, les processus cognitifs et mentaux responsables de l'expérience subjective, confrontés à un ou des miroirs eux-mêmes faisant partie de cette dernière. Cela pourrait impliquer une existence non pas permanente, mais épisodique du soi : nos processus cognitifs ne produisent pas en permanence une réflexion d'euxmêmes; or, en l'absence d'une "vision" que peut avoir l'expérience subjective d'elle-même - en l'absence d'une distance entre le soi et lui-même, on ne peut pas vraiment dire que ce dernier existe.

Cette analyse du problème du soi nous fournit les éléments de base nécessaires pour approfondir l'idée du langage intérieur comme outil capable d'expliciter à l'organisme le contenu de son expérience subjective.

Si le langage intérieur, en association avec l'environnement social, permet l'avènement et le maintien de la conscience de soi, c'est qu'il dédouble l'expérience subjective. Bien entendu, il ne la reproduit pas littéralement, en répliquant une pensée, une émotion ou une perception. Il en calque plutôt une représentation forcément différente de la réalité, au même titre qu'une réflexion en provenance d'un miroir constitue une représentation bidimensionnelle d'une réalité tridimensionnelle. Cette réflexion de l'expérience subjective se fait par le biais de mots, d'expressions verbales, grâce auxquels les divers éléments qui la constituent sont nommés et ordonnés. En nommant ces éléments, le langage intérieur se trouve à construire une représenta- 
tion schématique de choses autrement difficiles à distinguer les unes des autres: il en facilite l'identification. Et en favorisant l'identification de ce qui est vécu intérieurement, l'organisme (le soi) peut avec plus d'aisance ordonner ce vécu en classes d'événements pour former ainsi un tableau organisé d'autant plus facile à examiner. Ce tableau organisé est le concept de soi, une représentation plus ou moins complexe et sophistiquée qu'ont les gens d'eux-mêmes. ${ }^{20}$

Notons que ce recul par rapport à soi que permet le langage intérieur peut être aussi conçu sur un plan temporel. En nous situant par rapport à nos souvenirs et nos projets, notre identité prend du relief. En revanche, ne pas se décoller du moment présent appauvrit notre concept de soi, celui-ci étant réduit à un registre d'impressions subjectives immédiates.

Poussons maintenant notre analogie du miroir un peu plus loin.

Le langage intérieur, comme un miroir, est selon nous "étroit", "fini » dans l'espace; mais il est aussi "mobile». De ce fait, il peut être placé à différentes distances de l'objet réfléchi, et peut aussi en renvoyer plusieurs images variées dépendamment de l'angle qu'il présente par rapport à l'objet. On comprendra que plus il est «près» de l'expérience subjective, plus il en réfléchira un aspect particulier avec précision, et que plus il en est «loin", plus il la réfléchira dans son ensemble. Ce mouvement, combiné avec un angle spécifique par rapport, toujours, à l'expérience subjective, donne au langage intérieur une flexibilité importante qui en fait un miroir puissant.

En quoi, psychologiquement parlant, le langage intérieur peut-il être "amovible"? Le contenu du monologue intérieur, ce sur quoi l'organisme focalise son attention via le langage intérieur, représente à notre sens l'équivalent le plus juste au terme imagé "amovible". Il devrait en effet être facile de concevoir que l'individu qui se réfléchit à lui-même le contenu de son expérience subjective peut verbaliser intérieurement différents aspects de cette dernière : il peut se limiter à quelque chose d'immédiat et de saillant ( $*$ je déteste cette situation"), à quelque chose de global ("ce qui vient de m'arriver constitue définitivement un point tournant dans ma vie; tout ce que je ressens intérieurement à ce propos m'indique que..."), ou à quelque chose d'intermédiaire («cette rencontre inattendue a provoqué en moi $x$

20. R. L'Ecuyer (1978), Le concept de soi, Paris, Presses Universitaires de France. 
émotions, m'a rappelé $y$ souvenirs, et $\mathrm{m}$ 'a fait réfléchir sur $z, \ldots n)$. En fait, une gamme à peu près infinie de contenus du langage intérieur peut être imaginée entre ces deux extrêmes, représentant par analogie une distance plus ou moins prononcée du miroir par rapport à ce qui est observé. En d'autres termes, les propos qu'articule le langage intérieur peuvent réfléchir des aspects très variés et d'inégale importance par rapport à soi; ce contenu détermine ce qui est auto-observé.

Au moins deux idées peuvent être déduites suite à ce qui précède. Une première concerne les limites de la conscience de soi impliquées par notre analogie du miroir. Une personne placée en face d'un miroir ou manipulant une surface réfléchissante mobile ne parviendra jamais à observer toutes les parties de son corps - ne serait-ce que le dos de sa tête. Il s'ensuit, si on applique ce principe au langage intérieur ou à tout autre processus cognitif ayant la même fonction, qu'au moins certaines composantes de l'expérience subjective sont inévitablement appelées à rester à tout jamais dans l'ombre de soi. Si notre analogie est fondée, cela signifie que tout être vivant - aussi conscient soit-il de lui-même - est "victime" d'au moins quelques motivations ou valeurs inconscientes qui guident aveuglément certains de ses comportements.

Une seconde précision concerne le problème de la justesse des rapports verbaux. Les travaux de Nibett et Wilson ${ }^{21}$ nous rappellent que toute auto-observation (verbale ou autre) est sujette à des déformations, où l'analyse que fait une personne d'elle-même n'est pas toujours consistante avec ce qu'elle est vraiment, aux yeux d'autrui tout au moins ou telle qu'objectivée par des tests de personnalité, par exemple. Cet état de fait n'est pas particulièrement surprenant dans la mesure où le langage intérieur, en restructurant le vécu subjectif par le biais de symboles forcément différents de ce qu'il reflète, se trouve parfois à renvoyer des images relativement imprécises ou altérées de ce vécu. Dès qu'une réalité souvent non verbale est nommée et ordonnée arbitrairement, il est prévisible que des erreurs d'identification se glissent dans sa description. De plus, il ne faut pas confondre ce type d'erreurs avec la tendance persistante qu'ont les gens à se décrire comme ils voudraient être au lieu de comme ils sont. Le problème soulevé ici met encore en évidence la fragilité et les limites de la

21. R.E. Nibett et T.D. Wilson (1977), «Telling more than we can know : verbal reports on mental processes w, Psycbological Review, 3(84), 231-259. 
conscience de soi et du langage intérieur comme outil d'introspection; mais il n'implique pas, sous prétexte que les rapports verbaux sont parfois biaisés, qu'ils sont invalides.

\section{IV}

L'accent a été mis jusqu'à présent sur le rôle potentiel du langage intérieur comme un processus cognitif-clef impliqué dans l'avènement et le maintien de la conscience de soi. Nous postulons que ce processus est le plus apte à créer un espace intra-psychique nécessaire à un recul et à l'auto-observation, mais en aucun cas voulons-nous suggérer qu'il s'agit du seul processus cognitif capable d'opérer un tel recul par rapport à soi. Le lecteur conviendra certainement qu'il nous est tous déjà arrivé de réaliser qu'on était en train d'émettre tel ou tel comportement, ou qu'on se sentait gêné par exemple, tout en parlant avec une autre personne. D'où la constatation évidente qu'il est définitivement possible d'être conscient de soi à un moment donné sans qu'on ait à se parler à soimême à propos de soi - notre activité verbale étant à ce moment précis dirigée non pas vers soi mais vers autrui.

Le cas suivant ${ }^{22}$, ainsi que l'existence d'un concept de soi au moins rudimentaire chez certains primates (cette question sera abordée à la section V), met bien en évidence la possibilité pour un organisme d'être conscient de luimême en l'absence de langage intérieur.

E.B. est un homme de 54 ans chez qui un accident cérébro-vasculaire a eu comme effet d'élargir anormalement la portion frontale du ventricule latérale gauche ainsi que la section antérieure gauche de la fissure de Sylvius, congestionnant du même coup les entités anatomiques adjacentes. Une batterie de tests neuropsychologiques a confirmé une perte totale du langage in térieur chez le patient, malgré une préservation substantielle de l'expression écrite et de la compréhension du langage oral et écrit. Le niveau de conscience de soi de E.B. n'a pas été directement mesuré à l'aide d'un test approprié ${ }^{23}$, mais l'extrait suivant, tiré d'un

22. D.N. Levine, R. Calvanio et A. Popovics (1982), "Language in the absence of inner speech*, Neuropsychologia, 20, 391-409.

23. La reconnaissance de soi devant le miroir (dont il sera question plus loin dans cet article) pourrait etre un test approprié. Une autre stratégie serait d'administrer au patient l'Échelle de Conscience de Soi de Fenigstein et al. (1975), qui mesure la disposition à etre attentif à soi. A. Fenigstein, M.f. Scheier et A.H. Buss (1975), * Public and private self-consciousness : assessment and theory *, Journal of Consulting and Clinical Psycbology, 43, 522.527. 
rapport écrit par le patient à propos de son accident, est très révélateur:

J'ai commencé à voir tout en blanc et à entendre des voix et des sons et je pouvais voir ensuite des infirmières et un jeune docteur près de moi, qui me demandait mon nom. Je me sentais étourdi, engourdi et faible. J'essayais de bouger - sans succès; de parler - je n'émettais que des sons inarticulés. Plusieurs docteurs et infirmières m'examinaient, me posaient des questions (...) [citation : p. 394].

E.B., visiblement, était connaissant du contenu de son expérience subjective au moment de l'accident, malgré l'impossibilité pour lui de se parler à lui-même.

Cette constatation soulève la question suivante: quels autres processus cognitifs pourraient réfléchir au cerveau le contenu de son activité?

Deux candidats s'imposent ici : l'imagerie mentale 24 , puisqu'elle peut entre autres choses reproduire subjectivement des scènes fictives ou réelles mais passées dont l'acteur principal n'est autre que le sujet même qui voit ces images, et la mémoire, qui est capable de nous faire revivre des émotions, des pensées et des situations où le sujet de ces expériences passées est nous-mêmes. Dans ces deux cas, on retrouve les ingrédients nécessaires à la conscience de soi : la redondance (dédoublement de l'expérience subjective), la «distanciation», et par conséquent, le recul par rapport à soi. Nous nous devons toutefois d'insister sur le point suivant.

Ces deux processus ne peuvent généralement réfléchir à l'organisme que des comportements qu'il a émis ou qu'il envisage d'émettre. Ainsi, bon nombre d'éléments propres à l'identité personnelle (c'est-à-dire, nos valeurs, croyances religieuses et politiques, attitudes, etc. ${ }^{25}$ par exemple ne peuvent être reproduits visuellement (comment, en effet, *visualiser des attitudes ou des valeurs?). La mémoire peut certes ranimer des pensées ou des émotions passées, mais toujours, nous semble-t-il, par le biais de comportements remémorés; sa fonction * distanciatrice " emprunterait donc un chemin indirect. L'imagerie mentale et la mémoire peuvent donc représenter à l'organisme certaines facettes comporte-

24. Cf. M. Rollins (1989), Mental Imagery, New Haven \& London : Yale University Press, A. Morin et S. DeBlois (1989), "Gallup's mirrors : more than an operationalization of self-awareness in primates? *, Psychological Reports, 65, 287-291.

25. Cette definition de l'identité personnelle s'inspire de R. Puccetti (1969), « Brain transplantation and personal identity *, Analysis, 29(3), 65-77. 
mentales de son fonctionnement. Mais l'utilisation complémentaire du langage intérieur correspondrait de façon imagée à l'ajout d'un miroir beaucoup plus large, en mesure de réfléchir non seulement une image plus claire et complète de ce qui est vécu intérieurement, mais aussi capable de réfléchir une réalité qui en grande partie ne peut être représentée autrement qu'avec des mots. D'où la supériorité, selon nous, des mots (du langage intérieur) sur les images (l'imagerie mentale) pour la conscience de soi. Il est clair que l'imagerie mentale est capable de réfléchir à l'organisme certaines dimensions de lui-même que le langage intérieur est impuissant à "visualiser", notamment son image corporelle; mais on peut avoir accès à la même information en se décrivant verbalement à soi-même notre aspect physique.

Tout ceci nous permet de mieux comprendre comment E.B. pouvait avoir accès au contenu de son expérience subjective sans langage intérieur. On sait que ce patient possédait une capacité à l'imagerie mentale très supérieure à la moyenne : il avait suivi à l'âge de vingt ans un cours de lecture rapide qui l'encourageait à «voir» les mots au lieu de les «entendre». En d'autres termes, on lui avait enseigné dans ce cours à éviter de se répéter mentalement les mots écrits et de se les représenter plutôt sous forme d'images mentales. Cette stratégie a eu comme conséquence de favoriser chez E.B. le développement de son imagerie mentale au détriment de l'utilisation de son langage intérieur. D'où la précision suivante, proposée par ses neuropsychologues : "C'est purement une question de préférence terminologique si on prétend, comme nous l'avons fait, que notre patient était privé de langage intérieur au sens verbal, ou qu'il avait un langage intérieur, basé exclusivement sur l'imagerie mentale "26 Dans cette perspective, nous serions portés à croire que E.B. possède effectivement un mode de communication intra-psychique, empruntant des images mentales en guise de symboles, lui permettant peut-être même de visualiser la forme écrite d'un mot et de formuler ainsi des phrases cohérentes sans se "parler" à lui-même. Cela illustre bien jusqu'à quel point le cerveau peut adopter des stratégies cognitives alternatives pour parvenir à réfléchir sa propre activité. Il est possible d'ailleurs que les sourds-muets de naissance utilisent aussi ce genre de stratégies compensatoires, n'ayant jamais développé de langage in térieur verbal. 
Mais encore une fois, notre hypothèse postule une supériorité du langage intérieur sur tout autre processus cognitif impliqué dans la conscience de soi. Par conséquent, nous suggérons que E.B., aussi bien avant qu'après son accident cérébro-vasculaire, présentait un faible degré de conscience de soi par rapport à une population faisant un usage plus important du langage intérieur. Cette conjecture pourrait être vérifiée si une mesure sophistiquée de la conscience de $\operatorname{soi}^{27}$ était administrée chez une population de sourds-muets par exemple, dont les résultats seraient comparés à ceux obtenus auprès d'une population normale.

Comment déterminer expérimentalement l'existence d'une conscience de soi chez des organismes dans l'impossibilité de nous communiquer verbalement le contenu de leurs états mentaux? La psychologie comparative, incapable de répondre à cette question, a traditionnellement considéré ce problème comme étant irrésoluble par la science, jusqu'à ce que Gallup ${ }^{28}$ entre en scène. Grâce à l'utilisation d'une série de protocoles expérimentaux fort ingénieux ${ }^{29}$, ce chercheur a démontré que seuls les chimpanzés et les orangs-outans, mis à part l'humain, étaient capables de se reconnaîlre devant un miroir. Cette habileté représente une façon on ne peut plus directe de déterminer l'existence d'une conscience de soi (ainsi que d'un concept de soi au moins rudimentaire) chez tout organ isme visuellement compétent, puisque la reconnaissance de soi présuppose la possibilité de devenir l'objet de sa propre attention, et implique de la part de l'organisme qui se reconnaît une connaissance préalable tout au moins partielle de ce qu'il est.

Si on suppose ce raisonnement fondé, deux questions s'imposent ici. D'abord, cette conscience de soi chez le primate est-elle comparable à celle de l'humain, et si oui, cela signifie-t-il que le langage intérieur n'a rien à voir avec la conscience de soi (les singes étant vraisemblablement

27. Par exemple : l'Échelle de Conscience de Soi de Fenigstein et al. (op. cit.), cf., note 23.

28. Cf. Gallup, op. cit.; Gallup a publié pour la première fois les résultats de ses recherches au début des années 70 .

29. Il serait trop long de présenter ici le déroulement de ces expériences. Nous renvoyons le lecteur intéressé à G.G. Gallup Jr. (1977), "Self-recognition in primates : a comparative approach to the bidirectional properties of consciousness *, American Psycbologist, 32, 329-338. 
dépourvus de langage intérieur)? Ensuite, pourquoi seuls les chimpanzés et les orangs-outans, parmi tous les grands singes, possèdent-ils une conscience et un concept de soi?

À la première question, nous répondrons oui et non. Puisque la conscience de soi origine d'abord et avant tout de l'environnement social (idée à laquelle Gallup adhère), il faut croire que le monde social du chimpanzé et de l'orang-outan est assez complexe pour réfléchir à ces animaux l'essentiel de ce qu'ils sont : il leur permet vraisemblablement de prendre assez de recul face à eux-mêmes et de percevoir suffisamment de choses à leur propos pour saisir la réalité de leur existence. Donc, la conscience de soi du chimpanzé et de l'orang-outan est comparable à celle de l'humain dans le mécanisme responsable de son apparition.

Par contre, il est clair que l'humain possède une expérience subjective, un monde intérieur, beaucoup plus riche que les primates. Notamment, il ne partage pas avec ces derniers une identité personnelle (cf. p. 181), réalité psy. chologique nécessairement médiatisée par le langage. L'environnement social dont font partie le chimpanzé et l'orang-outan peut renvoyer à chacun de ses membres des informations relatives à leurs comportements, à leur image corporelle et à leur expérience subjective immédiate. Ainsi, lorsque ces animaux apprennent à se reconnaître dans un miroir, ils savent qui ils sont, leur environnement social leur fournissant tous les éléments nécessaires à cet effet. L'environnement social de l'humain renvoie aussi à chacun de ses membres toutes ces informations à son propos, sauf plusieurs composantes plus privées de son identité personnelle et de son monde intérieur en général, qu'il ne peut identifier qu'à travers une auto-analyse verbale serrée.

Donc, c'est cette qualité de l'expérience subjective humaine - dont certaines parties ne sont accessibles à l'individu que par l'introspection, et que le monde social, en tant que miroir, reste impuissant à refléter - qui distingue la conscience de soi lui étant associée de celle des animaux. On peut aussi comprendre dans cette perspective que si, effectivement, le langage intérieur n'a rien à voir avec la conscience de soi animale, il est probablement essentiel à la conscience de soi humaine.

Nous sommes maintenant en mesure de répondre à la deuxième question, en proposant simplement que le monde social des autres grands singes (dépourvus de la capacité de reconnaissance de soi) est trop pauvre en feedback pour 
leur permettre un recul suffisant par rapport à eux-mêmes. D'où une méconnaissance d'eux-mêmes, un concept de soi atrophié, et l'impossibilité conséquente d'inférer correctement l'identité de leur réflexion dans un miroir.

\section{VI}

En guise de conclusion à cet article, nous présentons diverses réflexions ayant trait au langage intérieur et à la conscience de soi, en relation avec la schizophrénie, la prière, la méditation, et les drogues psychodysleptiques.

Frith ${ }^{30}$ propose que les symptômes de la schizophrénie seraient le résultat d'une dissociation entre le will (le vouloir) et les actions émises par les patients. Il distingue entre eux deux types de symptômes chez le schizophrène: les signes * positifs * (subjectifs, non observables) comme les *délusions» et les hallucinations, où le patient a l'impression que des pensées qui ne lui appartiennent pas l'envahissent, et les signes "négatifs " (comportementaux, observables) comme le manque d'initiative, la pauvreté du langage, l'aplatissement émotionnel et le retrait social, où dans les cas extrêmes le patient cesse complètement d'agir ou de parler. Selon Frith, «(...) les expériences subjectives des schizophrènes (les signes positifs) sont la conséquence d'un manque de conscience des intentions du "vouloir" ce que nos plans et désirs nous suggèrent de faire (...); les signes négatifs représentent une détérioration encore plus sévère du même système, dont la conséquence est que les intentions du patient ne sont plus traduites en action .31 Notre propre vision de la conscience de soi et du rôle joué par le langage intérieur pour celle-ci nous porte à suggérer que le schizophrène est en fait capable d'observer ses états mentaux et ses comportements, mais ne parvient pas, pour une raison inconnue, à réaliser que ces choses lui appartiennent: il y aurait une dissociation entre les dimensions 1 et 2 de la conscience de soi - une dissociation entre l'identification d'éléments constituant son expérience subjective et la réalisation que cette expérience subjective est sienne. Ainsi, le patient se parlerait à lui-même, sans réaliser toutefois à certains moments que la voix interne qu'il entend est sienne, d'où son expérience d' * entendre des voix» et de

30. C.D. Frith (1987), *The positive and negative symptoms of shizophrenia reflect impairment in the perception and initiation of action *, Psychological Medecine, 17, 631-648.

31. Frith (op. cit.), pp. 638-640 
"recevoir des ordres" d'êtres qui contrôlent ses comportements et ses pensées de l'extérieur. Il n'y aurait pas «manque de conscience des intentions du vouloir $"$ mais bien une incapacité à comprendre que les intentions et états mentaux observés sont à soi ; et il serait peut-être plus juste de dire, au lieu de *les intentions du patient ne sont plus traduites en action", que le patient traduit (via le langage intérieur) des intentions en actions, souvent sans savoir que ces intentions sont siennes, et en ayant l'impression que ces commandes verbales viennent de l'extérieur.

Il vient tout juste d'être question de patients psychotiques qui, souvent, rapportent entendre des voix. Il est tentant ici d'avancer une idée fort provocante à propos de la prière. D'abord, notons que celle-ci s'articule incontestablement par le biais du langage intérieur; de plus, lorsque la prière prend une forme de communication active avec Dieu - lorsqu'elle est plus qu'une simple louange, beaucoup de gens prétendent recevoir des "réponses" à leurs *demandes": "Dieu me parle, me guide". Serionsnous alors aussi en présence de personnes qui, parfois, confondent leur propre voix interne avec celle d'un être supérieur (réel ou imaginé - cette distinction ne nous intéresse pas ici)? Jaynes ${ }^{32}$ répondrait probablement (avec nous) à cette question par l'affirmative, puisque selon cet auteur, l'homme primitif, lorsqu'il a commencé à se parler à lui-même, interprétait sa propre voix interne comme étant celle de dieux le guidant dans ses décisions.

Sur un autre plan d'idées, plusieurs auteurs ${ }^{33}$ prétendent que la méditation représente une pratique particulièrement efficace dans la recherche d'un degré de conscience de soi élevé. Cette suggestion nous semble insatisfaisante, compte tenu du fait que "méditer" est synonyme de "faire le vide» (taire sa voix interne), ce qui constitue précisément l'inverse d'une analyse de soi. D'ailleurs, «(...) la caractéristique la plus saillante propre à la plupart des états de conscience dits "mystiques" (ou de l'Orient) est précisément une absence d'un sens de soi... " ${ }^{34}$ Aucune recherche à notre connaissance relate par ailleurs des résultats relatifs à une supposée augmentation de conscience de soi suite à un entraînement à la méditation.

32. J. Jaynes (1986), "Consciousness and the voices of the mind", Canadian Psycbology/ Psycbologie Canadienne, 27(2), 128-139.

33. Dont Fenigstein et al. (op. cit.) et W.L. Mikulas (1986), « Self-control : essence and development . The psycbological Record, 36, 297-308.

34. J. DeWitt (op. cit.), p. 45. 
Il est reconnu que les usagers de drogues psychodysleptiques (hallucinogènes) rapportent fréquemment des expériences de distorsion de la conscience de soi, où l'individu, typiquement, a l'impression d'être à l'extérieur de lui-même et de se voir agir, ou éprouve le sentiment de comprendre avec beaucoup plus de clarté ce qu'il est et ce qu'il vit intérieurement. ${ }^{35}$ Comment, en quoi le L.S.D., la psylocybine ou le haschich par exemple, peuvent-ils augmenter temporairement le degré de conscience de soi du sujet intoxiqué? D'abord, il est possible que ces drogues, en favorisant l'introspection d'une façon mystérieuse, accentuent tout simplement l'usage du langage intérieur. Mais il est possible aussi que ces substances chimiques, du fait qu'elles modifient le fonctionnement du cerveau - et du même coup le cours normal de l'expérience subjective qu'il génère, confrontent l'usager à une expérience subjective déformée qu'il compare spontanément à ce qu'il vit in térieurement à jeun. Et de cette comparaison *expérience subjective normale-déformée * naîtrait l'opportunité pour l'individu d'observer ce qu'il est et ce qu'il vit habituellement sous un angle tout à fait nouveau. Donc, ici aussi, il y aurait dédoublement (expérience subjective normale-déformée), redondance, et conséquemment, recul par rapport à soi. Dans cette perspective, les anxiolytiques, les stimulants, les opiacés ou l'alcool, ne provoqueraient pas une augmentation substantielle du degré de conscience de soi, simplement parce que ces drogues déforment moins l'expérience subjective, ou affectent principalement des fonctions psychologiques inférieures.

Enfin, citons Ojemann ${ }^{36}$, qui est à notre connaissance un des seuls auteurs à établir avec nous un lien étroit entre le langage intérieur et la conscience de soi. Il nous dit, à propos des patients cérébro-lésés, que:

La réapparition de la conscience de soi chez un patient semble se faire parallèlement à la réapparition de son langage intérieur. Le langage intérieur peut être limité, embrouillé, restreint, anormal après une lésion cérébrale; mais au moins l'expérience consciente est revenue.

35. Cf. R. Mechouian (1973), Marijuana, New York : Academic Press. S. RadoucoThomas (1974), Pbarmacology, Toxicology and Abuse of Psychotomimetics. Québec : Les Presses de l'Université Laval.

36. G. Ojemann (1986), « Brain mechanisms for consciousness and conscious experience «, Canadian Psycbology/Psycbologie Canadienne, 27(2), 158-168 [Citation : p. 161]. 
Que très peu de chercheurs soulignent l'éventuel rapport de causalité pouvant exister entre le langage intérieur et la conscience de soi signifie peut-être que les idées développées à ce propos dans cet article constituent une élucubration monumentale; peut-être aussi sommes-nous sur une piste prometteuse négligée jusqu'à présent. Seule l'expérimentation en décidera.

École de Psychologie, Université Laval 